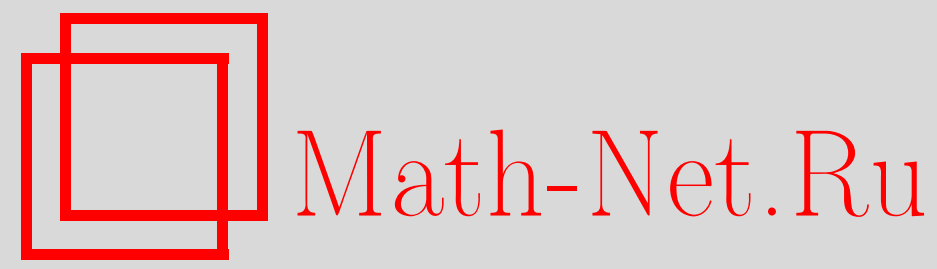

Н. Н. Вахания, Н. П. Канделаки, Случайные векторы со значениями в комплексных гильбертовых пространствах, Теория вероятн. и ее примен., 1996, том 41, выпуск 1, 31-52

DOI: https://doi.org/10.4213/tvp2772

Использование Общероссийского математического портала Math-Net.Ru подразумевает, что вы прочитали и согласны с пользовательским соглашением http://www . mathnet.ru/rus/agreement

Параметры загрузки:

IP: 3.81 .55 .215

26 апреля 2023 г., 14:57:47

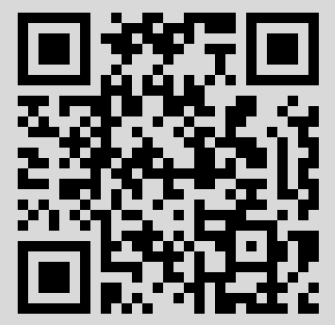




\title{
СЛУЧАЙНЫЕ ВЕКТОРЫ СО ЗНАЧЕНИЯМИ В КОМПЛЕКСНЫХ ГИЛЬБЕРТОВЫХ ПРОСТРАНСТВАХ ${ }^{1)}$
}

\begin{abstract}
Предпринята попытка систематического подхода к изучению некоторых основных вероятностных понятий применительно $\mathrm{k}$ случайному вектору со значениями в комплексном гильбертовом пространстве. Такому случайному вектору можно естественным образом сопоставить пару случайных векторов со значениями в соответствующем гильбертовом пространстве, и это дает другой возможный подход $\mathbf{~}$ описанию комплексных векторов. Относительно вопроса измеримости и понятия математического ожидания эти два подхода по существу являются одинаковыми. Однако относительно вопросов, связанных с ковариационным оператором, дело обстоит иначе. Ключевым понятием работы является правильный случайный вектор, т.е. такой вектор, для которого указанные два подхода приводят к одним и тем же результатам также и в вопросах, связанных с ковариационным оператором. Изучается круг вопросов, возникахших в связи с понятием правильности случайного вектора.
\end{abstract}

Клточевые слова и фразы: комплексная гауссовская случайная величина, случайный вектор в комплексном гильбертовом пространстве, правильное разложение, правильный случайный вектор, гауссовский случайный вектор в узком и широком смысле, шёнберговская мера, почти наверное ортогональность случайных векторов.

\section{Введение}

Комплексные случайные величины и векторы появляются в основном в теории стационарных случайных процессов и за пределами этой теории редко встречаются. По-видимому, этим можно объяснить тот факт, что в комплексном случае нет полной ясности и однозначности в отношении такого важного для всей теории вероятностей понятия, как гауссовское распределение. Так же обстоит дело и с понятием ковариационного оператора. Причина неоднозначности в обоих случаях одна и та же: при одном подходе комплексная случайная величина $\zeta=\zeta^{\prime}+i \zeta^{\prime \prime}$ рассматривается как двумерная, $\left(\zeta^{\prime}, \zeta^{\prime \prime}\right)$, со значениями в вещественной

*Институт вычислительной математики им. Н. И. Мусхелишвили АН Грузии, ул. Акурсках, 8, 380093 Тбилиси, Грузия.

1) Настоящее исследонание стало возможным частично благодаря поддержке Международного научного фонда, грант № MXC000. 
плоскости $\mathbf{R}^{2}$, а при другом - как одномерная в комплексной плоскости $\mathbf{C}^{1}$. При первом, широком, подходе случайная величина $\zeta$ называется гауссовской, если пара $\left(\zeta^{\prime}, \zeta^{\prime \prime}\right)$ просто имеет гауссовское распределение в $\mathbf{R}^{2}$. А при другом, узком, подходе дополнительно требуется взаимная независимость $\zeta^{\prime}$ и $\zeta^{\prime \prime}$ и равенство их дисперсий; появление дополнительных условий можно объяснить как естественное следствие этого (комплексного) подхода. Большинство авторов (К. Ито, Ж. Невё, Ю. А. Розанов, Т. Хида) придерживается этого более узкого определения, хотя некоторые авторы, (например, Г. Крамер и М. Лидбеттер в кните «Стационарные случайные процессы») используют широкое определение. Эта неоднозначность в определении гауссовской случайной величины вызывает соответствуюшую неоднозначность в определении гауссовских случайных векторов в конечномерных и, особенно, бесконечномерных комплексных пространствах. Авторы настоящей работы отдают предпочтение узкому определению, использующему ко̆мплексную структуру пространства. Однако часть работы посвящена сравнению этих двух подходов и поэтому мы используем прилагательные «узкий» и «широкий».

Ключевым понятием настоящей работы является правильный случайный вектор. Так мы называем случайный вектор, для которого вещественный и комплексный подходы приводят к одним и тем же результатам. В частности, гауссовские в широком смысле правильные случайные векторы являются гауссовскими и в узком смысле.

Параграф 1 носит подготовительный характер. Обсуждаются простые и естественные понятия правильного разложения элемента комплексного гильбертова пространства $H$ и правильного оператора в прямой сумме двух вещественных гильбертовых пространств.

В § 2 на базе этих предварительных понятий и фактов вводится и подробно анализируется понятие правильного случайного вектора. Здесь же вводится класс случайных векторов, распределения которых обладают определенными свойствами симметрии (слабо изометрически инвариантны). Имея в виду сравнение с правильными векторами, такие случайные векторы мы условно называем суперправильными, хотя классы случайных векторов с близкими свойствами симметрии для конечномерного случая изучались в литературе под различными названиями (сферические, изотропные).

В §3 продолжается изучение правильных и суперправильных случайных векторов. Вводится и обсуждается понятие гауссовского случайного вектора в узком и широком смысле.

Параграф 4 посвящен вопросу почти наверное взаимной ортогональности случайных векторов. Понятие правильности оказалось существенным и в этом вопросе. Также значительной оказалась в этом вопросе разница между вещественным и комплексным случаями. Показывается, что в комплексном гильбертовом пространстве $H$ компоненты правиль- 
ной пары случайных векторов с линейным носителем могут быть почти наверное взаимно ортогональны лишь в том тривиальном случае, когда они почти наверное принадлежат взаимно ортогональным подпространствам пространства $H$. В качестве следствия получаем, что в комплексном случае, в отличие от вешественного случая, компоненты гауссовской пары случайных векторов могут быть взаимно ортогональны с вероятностью 1 лишь в указанном выше тривиальном случае.

В § 5 вводится и изучается один специальный класс суперправильных случайных векторов, именно, случайные линейные образы $\Theta \xi$ гауссовских случайных векторов $\xi$ в $H$ в предположении взаимной независимости $\Theta$ и $\xi$. В частном случае, когда случайный оператор $\Theta$ скалярно пропорционален тождественному оператору в $H$, получаются распределения, которые тесно связаны с известной теоремой Шёнберга о представлении всех непрерывных изометрически инвариантных положительно определенных функционалов в вещественном гильбертовом пространстве. Распределения, соответствуюшие этому частному случаю, изучались в литературе вне связи с теоремой Шёнберга под названием эллиптически контуированных мер. Большая часть $§ 5$ посвящена изучению обобщенных шёнберговских мер, т.е. мер, соответствуюгих общему случаю, когда $\Theta$ - произвольный случайный линейный отератор, не зависящий от $\xi$.

В целом, работа представляет собой попытку систематическото подхода к некоторым основным аспектам теории вероятностных распределений в комплексных гильбертовых пространствах. Заметим, что аналогичный подход, основанный на соответствующей модификации понятия правильного случайного вектора, возможен и в теории вероятностных распределений в гильбертовых пространствах над телом кватернионов.

Мы выражаем признательность Т. Л. Чантладзе за полезные обсуждения с ним отдельных частей настоящей работы.

\section{§1. Основные предварительные понятия и результаты}

Пусть $H$ - сепарабельное комплексное гильбертово пространство. Выберем в $H$ произвольный ортонормированный базис и обозначим через $H_{\mathbf{R}}$ совокупность всех тех элементов пространства $H$, которые имеют вещественные коэффициенты Фурье в разложении по этому базису. $H_{\mathbf{R}}$, разумеется, зависит от выбора базиса, однако в обозначении эта зависимость не отражена, так как базис всегда будет считаться зафиксированным. Пусть $c_{1}, c_{2}, \ldots$ - коэффициенты Фурье вектора $h \in H$ в выбранном базисе. Полагая $c_{k}=c_{k}^{\prime}+i c_{k}^{\prime \prime}$ с вещественными $c_{k}^{\prime}$ и $c_{k}^{\prime \prime}$, получим разложение произвольного элемента $h \in H$ в виде $h=h^{\prime}+i h^{\prime \prime}$, где $h^{\prime}$ и $h^{\prime \prime}$ принадлежит $H_{\mathbf{R}}$. Разложение $h=u+i v$ будем называть правильным, если $u ; v \in H_{\mathbf{R}}$. Понятие правильности разложения зависит oт пространства $H_{\mathbf{R}}$ и, следовательно, от выбора базиса в $H$ (в одномерном случае $H=\mathbf{C}^{1}$ естественное разложение комплексного числа $\lambda$

2 Теория вероятностей и ее применения, № 1 
в виде $\lambda=\operatorname{Re} \lambda+i \operatorname{Im} \lambda$ является правильным только относительно базиса $e=1$ ). В фиксированном базисе правильное разложение, очевидно, единственно.

Совокупность $H_{\mathbf{R}}$ представляет собой аддитивную группу и замкнута относительно умножения на вещественные числа и перехода к пределу. Кроме того, скалярные произведения векторов из $\boldsymbol{H}_{\mathbf{R}}$ принимают только вещественные значения. Поэтому, ограничив поле скаляров вещественными числами, можем считать $H_{\mathbf{R}}$ вещественным гильбертовым пространством. Таким образом, соотношением $h=h^{\prime}+i h^{\prime \prime} \longleftrightarrow$ $\left(h^{\prime}, h^{\prime \prime}\right)=\widehat{h}$, где $h^{\prime}+i h^{\prime \prime}$ есть правильное разложение элемента $h$, a $\widehat{h}$ обозначает пару $\left(h^{\prime}, h^{\prime \prime}\right)$, комплексное пространство $H$ отображается взаимно однозначно и с сохранением операций сложения и умножения на вепественные скаляры на вещественное линейное пространство $H_{\mathbf{R}} \times H_{\mathbf{R}}$, которое является гильбертовым пространством относительно скалярного произведения

$$
\langle\widehat{h} \mid \widehat{g}\rangle=\left(h^{\prime} \mid g^{\prime}\right)+\left(h^{\prime \prime} \mid g^{\prime \prime}\right)
$$

где $(\cdot \mid \cdot)$ - скалярное произведение в $H$. Это гильбертово пространство называется обычно гильбертовой прямой суммой двух гильбертовых пространств $H_{\mathbf{R}}$ и обозначается $H_{\mathbf{R}} \oplus H_{\mathbf{R}}$. Очевидно, что если в исходном пространстве $H$ допустить умножение только на вешественные скаляры и ввести новое скалярное произведение $(h \mid g)_{0}=\operatorname{Re}(h \mid g)$, то получим вещественное гильбертово пространство $H_{0}$, которое, по существу, совпадает с $H_{\mathbf{R}} \oplus H_{\mathbf{R}}$ в том смысле, что изометрически изоморфно ему. Пространства же $H_{0}$ и $H$, хотя и состоят из одних и тех же элементов и изоморфны друг другу как аддитивные группы, и гомеоморфны как топологические пространства, отличаются друг от друга как гильбертовы пространства. Заметим, например, что для любого $x \in H_{\mathbf{R}} \subset H$ векторы $x$ и $i x$ пропорциональны в $H$, а в $H_{0}$ они линейно независимы и даже взаимно ортогональны. Это различие вызывает такое же различие между пространствами $H$ и $H_{\mathbf{R}} \oplus H_{\mathbf{R}}$. Оно связано, конечно, с ограничением поля скаляров.

Любой ограниченный линейный оператор $A$ в комплексном гильбертовом пространстве $H$ порождает соответствующий линейный ограниченный оператор $\widehat{A}$ в вещественном гильбертовом пространстве $H_{\mathbf{R}} \oplus$ $H_{\mathbf{R}}$. Oператор $\widehat{A}$ соответствует оператору $A$ в том смысле, что если $g=A h$, то $\widehat{g}=\widehat{A} \hat{h}$. Найдем это соответствие между $A$ и $\widehat{A}$. Любой линейный оператор в $H_{\mathbf{R}} \oplus H_{\mathbf{R}}$ можно записать как $(2 \times 2)$-матрицу элементами которой являются операторы из $H_{\mathbf{R}}$ в $H_{\mathbf{R}}$. Мы найдем матригу $\left\|\widehat{A}_{i j}\right\|$ оператора $\hat{A}$, соответствующего оператору $A$. Для любого $h \in H$ имеем $A h=A h^{\prime}+i A h^{\prime \prime}$. Беря правильные разложения элементов $A h^{\prime}$ и $A h^{\prime \prime}$, получим: $A h=\left(A h^{\prime}\right)^{\prime}-\left(A h^{\prime \prime}\right)^{\prime \prime}+i\left[\left(A h^{\prime}\right)^{\prime \prime}+\left(A h^{\prime \prime}\right)^{\prime}\right]$. С другой стороны, $A h=\left(A h^{\prime}\right)+i\left(A h^{\prime}\right)^{\prime \prime}$. Поэтому для пюбого $x \subset H_{\mathbf{R}}$ имеем 
соотношения

$$
\widehat{A}_{11} x=\widehat{A}_{22} x=(A x)^{\prime}, \quad-\widehat{A}_{12} x=\widehat{A}_{21} x=(A x)^{\prime \prime} .
$$

Следовательно, матрица оператора $\widehat{A}$ имеет специальный вид: $\widehat{A}_{11}=$ $\widehat{A}_{22}, \widehat{A}_{12}=-\widehat{A}_{21}$. Линейные операторы в $H_{\mathbf{R}} \oplus H_{\mathbf{R}}$ с такими матрицами будем называть комплексно правильными, или просто правильными операторами. Матрицы правильных операторов обладают свойством перестановочности с матрицей

$$
J=\left\|\begin{array}{cc}
0 & -1 \\
1 & 0
\end{array}\right\|,
$$

соответствующей оператору умножения на мнимую единицу $i$ ( 0 и 1 обозначают нулевой и единичный оператор соответственно). Это свойство характеризует класс правильных операторов в $H_{\mathbf{R}} \oplus H_{\mathbf{R}}-$ только таким операторам соответствуют линейные операторы в $H$, в других случаях у соответствуюших операторов в $H$ нет свойства однородности относительно всех комплексных скаляров (свойства однородности относительно вешественных скаляров, аддитивности и непрерывности сохраняются). Нетрудно видеть, что данному правильному оператору $\left\|\widehat{A}_{i j}\right\|$ в $H_{\mathbf{R}} \oplus H_{\mathbf{R}}$ соответствует в $H$ оператор $A=A^{\prime}+i A^{\prime \prime}$, где $A^{\prime}$ и $A^{\prime \prime}$ принадлежат $L(H)$ и на $H_{\mathbf{R}}$ имеем: $A^{\prime}=\widehat{A}_{11}=\widehat{A}_{22}$ и $A^{\prime \prime}=-\widehat{A}_{12}=\widehat{A}_{21}$. В качестве следствия этого соответствия между операторами из $L(H)$ и $L\left(H_{\mathbf{R}} \oplus H_{\mathbf{R}}\right)$ получаем следующее утверждение: любой линейный оператор $A \in L(H)$ допускает разложение $A=A^{\prime}+i A^{\prime \prime}$, которое правильно в том смысле, что оба оператора $A^{\prime}$ и $A^{\prime \prime}$ оставляют инвариантной совокупность $H_{\mathbf{R}} \subset H$. Нетрудно показать также, что такое разложение единственно.

Совокупность всех правильных операторов составляет банахову подалгебру банаховой алгебры $L\left(H_{\mathbf{R}} \oplus H_{\mathbf{R}}\right)$ всех линейных ограниченных операторов $\left\{\right.$ вешественном пространстве $H_{\mathbf{R}} \oplus H_{\mathbf{R}}$, и эта подалгебра, по существу, совпадает с банаховой алгеброй $L(H)$ всех линейных ограниченных операторов в комплексном пространстве $H$.

Пусть $(\Omega, \mathcal{A}, \mathbf{P})$ - некоторое основное вероятностное пространство. Любая функция $\xi: \Omega \rightarrow H$ допускает разложение $\xi(\omega)=\xi^{\prime}(\omega)+i \xi^{\prime \prime}(\omega)$, правильное при каждом $\omega \in \Omega$. Измеримость функции $\xi$ равносильна измеримости пары функций $\xi^{\prime}$ и $\xi^{\prime \prime}$, т.е. измеримости функции $\Omega \rightarrow$ $H_{\mathbf{R}} \oplus H_{\mathbf{R}}$, огределяемой соотношением $\omega \longmapsto\left(\xi^{\prime}(\omega), \xi^{\prime \prime}(\omega)\right)=\hat{\xi}(\omega)$. При этом, как и для вещественных пространств, ввиду сепарабельности $H$ сильная и слабая измеримости эквивалентны (более подробно об этом см. в $[1$, гл. $1, \S 2])$. Случайным вектором (или элементом) со значениями в комплексном гильбертовом пространстве $H$ естественно называть функцию $\xi=\xi^{\prime}+i \xi^{\prime \prime}$, для которой функция $\hat{\xi}=\left(\xi^{\prime}, \xi^{\prime \prime}\right)$ является случайным вектором (элементом) со значениями в вешественном гильбертовом 
пространстве $H_{\mathbf{R}} \oplus H_{\mathbf{R}}$ (это равносильно тому, что $\xi^{\prime}$ и $\xi^{\prime \prime}$ являются случайными векторами в $H_{\mathbf{R}}$ ).

Мы будем рассматривать только такие случайные векторы $\xi$, которые имеют слабый второй порядок, т.е. для каждого $h \in H$ удовлетворяют условию $\mathbf{E}|(\xi \mid h)|^{2}<\infty$. Это условие, очевидно, равносильно условию существования слабого второго порядка для обоих случайных векторов $\xi^{\prime}$ и $\xi^{\prime \prime}$.

Легко проверить также, что математическое ожидание (среднее) $\mathbf{E} \xi$ случайного вектора $\xi$, которое можно определить, как обычно, с помошью интеграла Петтиса (т.е. соотношением $(\mathbf{E} \xi \mid h)=\mathbf{E}(\xi \mid h), h \in H)$, равно $\mathbf{E} \xi=\mathbf{E} \xi^{\prime}+i \mathbf{E} \xi^{\prime \prime}$. Это есть правильное разложение вектора $\mathbf{E} \xi \in H$, и поэтому в прямой сумме $H_{\mathbf{R}} \oplus H_{\mathbf{R}}$ этому вектору соответствует паpa $\left(\mathbf{E} \xi^{\prime}, \mathbf{E} \xi^{\prime \prime}\right)$. В самом деле, для любого вектора $h=h^{\prime}+i h^{\prime \prime} \in H$ $\left(h^{\prime}, h^{\prime \prime} \in H_{\mathbf{R}}\right)$ имеем равенство

$$
\mathbf{E}(\xi \mid h)=\mathbf{E}\left(\xi^{\prime} \mid h^{\prime}\right)+\mathbf{E}\left(\xi^{\prime \prime} \mid h^{\prime \prime}\right)+i\left[\mathbf{E}\left(\xi^{\prime \prime} \mid h^{\prime}\right)-\mathbf{E}\left(\xi^{\prime} \mid h^{\prime \prime}\right)\right] .
$$

$\mathrm{C}$ другой стороны, обозначая $\mathbf{E} \xi$ через $m$ и беря правильное разложение $m=m^{\prime}+i m^{\prime \prime}$, получим равенство

$$
(m \mid h)=\left(m^{\prime} \mid h^{\prime}\right)+\left(m^{\prime \prime} \mid h^{\prime \prime}\right)+i\left[\left(m^{\prime \prime} \mid h^{\prime}\right)-\left(m^{\prime} \mid h^{\prime \prime}\right)\right] .
$$

Все скалярные произведения в правой части равенства (3) вещественны, так как принятые разложения для $\xi$ и $h$ правильные. Далее, в силу определения математического ожидания (интеграла Петтиса) левые части равенств (3) и (4) равны при всех $h \in H$. Отсюда следуют нужные равенства $\mathbf{E} \xi^{\prime}=m^{\prime}, \mathbf{E} \xi^{\prime \prime}=m^{\prime \prime}$ и $\mathbf{E} \xi=\mathbf{E} \xi^{\prime}+i \mathbf{E} \xi^{\prime \prime}$.

Таким образом, математическому ожиданию случайного вектора $\xi$ соответствует математическое ожидание пары $\left(\xi^{\prime}, \xi^{\prime \prime}\right)$, соответствующей вектору $\xi$. В этом смысле можем написать $\widehat{\mathbf{E}} \xi=\mathbf{E}\left(\xi^{\prime}, \xi^{\prime \prime}\right)$ и сказать, что относительно математического ожидания соответствие между пространствами $H$ и $H_{\mathbf{R}} \oplus H_{\mathbf{R}}$ правильное. Относительно ковариационного оператора, однако, дело обстоит несколько иначе. Два подхода, основанные на рассмотрении $\xi$ как случайного вектора в комплексном гильбертовом пространстве $H$ и как случайного вектора $\left(\xi^{\prime}, \xi^{\prime \prime}\right)$ в вещественном гильбертовом пространстве $H_{\mathbf{R}} \oplus H_{\mathbf{R}}$, не всегда приводят к одному и тому же определению.

\section{§2. Правильные и суперправильные случайные векторы}

Ковариапионный оператор $K_{\xi}$ случайного вектора $\xi$ в комплексном гильбертовом пространстве $H$ естественно определить по аналогии с вешественным случаем равенством

$$
\left(K_{\xi} h \mid h\right)=\mathbf{E}|(\xi-\mathbf{E} \xi \mid h)|^{2}, \quad h \in H .
$$


Определенный таким образом оператор есть линейный ограниченный самосопряженный положительный оператор из $H$ в $H$. Заметим, что самосопряженный оператор мы называем положительным, если все значения соответствующей квадратичной формы неотрицательны. Элементарные вычисления дают равенство

$$
\left(K_{\xi} h \mid h\right)=\left(B h^{\prime} \mid h^{\prime}\right)+\left(B h^{\prime \prime} \mid h^{\prime \prime}\right)+2\left(\left(T_{\xi^{\prime} \xi^{\prime \prime}}-T_{\xi^{\prime} \xi^{\prime \prime}}^{*}\right) h^{\prime} \mid h^{\prime \prime}\right),
$$

где $B=B_{\xi^{\prime}}+B_{\xi^{\prime \prime}}$ и $B_{\xi^{\prime}}$ и $T_{\xi^{\prime} \xi^{\prime \prime}}$ есть, соответственно, ковариационный оператор случайного вектора $\xi^{\prime}$ и оператор взаимной ковариации $\xi^{\prime}$ и $\xi^{\prime \prime}$, которые определяются следуюшими равенствами (более подробную информацию об этих понятиях, включая комплексный случай, можно получить в $[1$, гл. $3, \S 2])$

$$
\begin{aligned}
\left(B_{\xi^{\prime}} x \mid x\right) & =\mathbf{E}\left(\xi^{\prime}-\mathbf{E} \xi^{\prime} \mid x\right)^{2} \\
\left(T_{\xi^{\prime} \xi^{\prime \prime}} x \mid y\right) & =\mathbf{E}\left(\xi^{\prime}-\mathbf{E} \xi^{\prime} \mid x\right)\left(\xi^{\prime \prime}-\mathbf{E} \xi^{\prime \prime} \mid y\right), \quad x, y \in H_{\mathbf{R}} .
\end{aligned}
$$

Равенство $(6)$ показывает, что $(2 \times 2)$-матрица оператора $\widehat{K}_{\xi}: H_{\mathbf{R}} \oplus$ $H_{\mathbf{R}} \longrightarrow H_{\mathbf{R}} \oplus H_{\mathbf{R}}$, соответствуюшего оператору $K_{\xi}: H \rightarrow H$, имеет вид

$$
\widehat{K}_{\xi}=\left\|\begin{array}{cc}
B & -2 T_{\xi^{\prime} \xi^{\prime \prime}} \\
2 T_{\xi^{\prime} \xi^{\prime \prime}} & B
\end{array}\right\|
$$

Ковариационный оператор $K_{\hat{\xi}}=K_{\left(\xi^{\prime}, \xi^{\prime \prime}\right)}$ в гильбертовой прямой сумме $H_{\mathbf{R}} \oplus H_{\mathbf{R}}$, согласно данному выше обшему определению ковариационного оператора в вешественном гильбертовом пространстве, определяется равенством $\left\langle K_{\left(\xi^{\prime}, \xi^{\prime \prime}\right)} \widehat{h} \mid \widehat{h}\right\rangle=\mathbf{E}\langle\hat{\xi}-\mathbf{E} \hat{\xi} \mid \widehat{h}\rangle^{2}$, где $\mathbf{E} \hat{\xi}=\left(\mathbf{E} \xi^{\prime}, \mathbf{E} \xi^{\prime \prime}\right)$, $\hat{\xi}=\left(\xi^{\prime}, \xi^{\prime \prime}\right)$ и $\hat{h}=\left(h^{\prime}, h^{\prime \prime}\right)$, а скалярное произведение $\langle\cdot \mid \cdot\rangle$ определяется равенством (1). Отсюда, как легко видеть, получаем

$$
K_{\widehat{\xi}}=K_{\left(\xi^{\prime}, \xi^{\prime \prime}\right)}=\left\|\begin{array}{cc}
B_{\xi^{\prime}} & T_{\xi^{\prime} \xi^{\prime \prime}}^{*} \\
T_{\xi^{\prime} \xi^{\prime \prime}} & B_{\xi^{\prime \prime}}
\end{array}\right\|
$$

Как было отмечено выше, только правильным линейным операторам в $H_{\mathbf{R}} \oplus H_{\mathbf{R}}$ соответствуют линейные операторы в $H$. Поэтому оператору $K_{\left(\xi^{\prime}, \xi^{\prime \prime}\right)}$ соответствует линейный огератор в $H$ лишь в том случае, если он правильный оператор, т.е. если $B_{\xi^{\prime}}=B_{\xi^{\prime \prime}}$ и $T_{\xi^{\prime} \xi^{\prime \prime}}^{*}=-T_{\xi^{\prime} \xi^{\prime \prime}}$. Случайный вектор со значениями в $H_{\mathbf{R}} \oplus H_{\mathbf{R}}$ будем называть правильным, если он имеет правильный ковариационный оператор $K_{\hat{\xi}}$ (оператор $\hat{K}_{\xi}$, соответствуюший ковариационному оператору $K_{\xi}$, разумеется, всегда правильный, как и любой оператор в $H_{\mathbf{R}} \oplus H_{\mathbf{R}}$, соответствующий линейному оператору в $H$ ). Случайный вектор $\xi$ со значениями в $H$ будем называть правильным, если соответствующий ему случайный вектор $\left(\xi^{\prime}, \xi^{\prime \prime}\right)$ - правильный в $H_{\mathbf{R}} \oplus H_{\mathbf{R}}$. Следующая теорема, доказательство которой можно провести непосредственными вычислениями, 
дает несколько эквивалентных между собой характеризаций правильности случайных векторов в $H$.

Теорема 1. Пусть $\xi-$ случайный вектор со слабым вторьлм порядком в сепарабельном комплексном аильбертовом пространстве $H$. Зафиксируем некоторый ортонормированныи базис в $H$ и представим $\xi$ в виде правильного разложения: $\xi=\xi^{\prime}+i \xi^{\prime \prime}\left(\xi^{\prime}, \xi^{\prime \prime} \in H_{\mathbf{R}}\right)$. Следующие условия эквивалентны ( к каждое из них выражает свойство правильности случайного вектора $\xi)$ :

(1) $K_{\hat{\xi}}=K_{\left(\xi^{\prime}, \xi^{\prime \prime}\right)}$ ecmь правильный оператор в $H_{\mathbf{R}} \oplus H_{\mathbf{R}}$, m.e. $B_{\xi^{\prime}}=$ $B_{\xi^{\prime \prime}} u T_{\xi^{\prime} \xi^{\prime \prime}}^{*}=-T_{\xi^{\prime} \xi^{\prime \prime}}$;

(2) $K_{\hat{\xi}}=\frac{1}{2} \widehat{K}_{\xi}($ cM. (9) $u(8))$;

(3) $K_{\left(\xi^{\prime}, \xi^{\prime \prime}\right)} J=J K_{\left(\xi^{\prime}, \xi^{\prime \prime}\right)}($ c.⿲. (2) $u(9))$;

(4) $\mathbf{E}[\operatorname{Re}(\xi \mid h) \operatorname{Im}(\xi \mid h)]=0$ для каждого $h \in H$;

(5) $\mathbf{E}[\operatorname{Re}(\xi \mid h)]^{2}=\mathrm{E}[\operatorname{Im}(\xi \mid h)]^{2}$ для каждого $h \in H$;

(6) $\mathbf{E}(\xi \mid h)^{2}=0$ для каждого $h \in H$.

3 а м е ч а н е 1. Существует бесконечное множество случайных векторов $\xi$, которые имеют один и тот же ковариационный оператор $K_{\xi}$ (и поэтому один и тот же $\widehat{K}_{\xi}$ ), но для которых ковариационные операторы $K_{\left(\xi^{\prime}, \xi^{\prime \prime}\right)}$ все различны.

3 а м е ч а н и е 2. Теорема 1, в частности, показывает, что, хотя разложение вектора зависит от выбора базиса, правильность случайного вектора есть свойство его распределения и не зависит от базиса.

3 а м е ч а н и е 3 . В одномерном случае $\left(H=\mathrm{C}^{1}\right)$ правильность случайной величины означает некоррелированность ее вещественной и мнимой частей и равенство их дисперсий (это согласуется с общим определением, так как в одномерном случае равенство $T^{*}=-T$ означает, что $T=0)$.

Ясно также, что правильность случайного вектора $\xi$ в общем (многомерном) случае означает его правильность в слабом смысле, т.е. правильность всех одномерных (комплексных) случайных величин $(\xi \mid h)$, $h \in H$.

Напомним, что гильбертова прямая сумма $H \oplus H$ двух комплексных гильбертовых пространств $H$ определяется как комплексное линейное пространство всех пар $\left(h_{1}, h_{2}\right)$ векторов из $H$ с естественным (покоординатным) определением комплексных линейных операций и со скалярным произведением, определяемым следующей формулой, аналогичной (1):

$$
\left\langle\left(h_{1}, h_{2}\right) \mid\left(g_{1}, g_{2}\right)\right\rangle=\left(h_{1} \mid g_{1}\right)+\left(h_{2} \mid g_{2}\right) \text {. }
$$

Используя это равенство и один из критериев теоремы 1 можно доказать непосредственным вычислением следующее утверждение.

Теорема 2. Если $\xi$ и $\eta-$ случайные векторы в комплексном гильбертовом пространстве $H$, то случайный вектор $(\xi, \eta)$ в гильбертовой прямой сумме $H \oplus H$ является правильньм в том и только том случае, 
если $\xi$ и $\eta$ оба правильны и выполнены также условия (см. (7))

$$
T_{\xi^{\prime} \eta^{\prime}}=T_{\xi^{\prime \prime} \eta^{\prime \prime}}, \quad T_{\xi^{\prime} \eta^{\prime \prime}}=-T_{\xi^{\prime \prime} \eta^{\prime}}
$$

Пусть $T_{\xi \eta}$ обозначает оператор взаимной ковариащии $\xi$ и $\eta$, который определяется соотношением (определение, данное в (7), относится к случаю вешественных пространств):

$$
\left(T_{\xi \eta} h \mid g\right)=\mathbf{E}(\xi-\mathbf{E} \xi \mid h) \overline{(\eta-\mathbf{E} \eta \mid g)}, \quad h, g \in H
$$

Нетрудно показать также прямым вычислением, что случайные векторы $\xi$ и $\eta$ некоррелированны, т.е. $T_{\xi \eta}=0$ в том и только в том случае, если $T_{\xi^{\prime} \eta^{\prime}}=-T_{\xi^{\prime \prime} \eta^{\prime \prime}}, T_{\xi^{\prime} \eta^{\prime \prime}}=T_{\xi^{\prime \prime} \eta^{\prime}}$. Поэтому в качестве следствия теоремы 2 получаем следующее утверждение.

Теорема 3. Компоненты $\xi$ и $\eta$ правильного случайного вектора $(\xi, \eta)$ некоррелированны $\left(T_{\xi \eta}=0\right)$ в том и только том случае, если выполнены условия:

$$
T_{\xi^{\prime} \eta^{\prime}}=-T_{\xi^{\prime \prime} \eta^{\prime \prime}}=T_{\xi^{\prime} \eta^{\prime \prime}}=T_{\xi^{\prime \prime} \eta^{\prime}}=0
$$

3 а м е ч ан и е. Существуют даже правильные случайные векторы $\xi$ и $\eta$, которые некоррелированны $\left(T_{\xi \eta}=0\right)$, но для которых условие теоремы 3 не выполняется. Вот простой пример: $\xi=\xi^{\prime}+i \xi^{\prime \prime}, \eta=\xi^{\prime \prime}+i \xi^{\prime}$, $\xi^{\prime}$ и $\xi^{\prime \prime}$ взаимно независимы и $\mathbf{E} \xi^{\prime}=\mathbf{E} \xi^{\prime \prime}=0, B_{\xi^{\prime}}=B_{\xi^{\prime \prime}}$.

Теперь мы введем другое свойство случайных векторов, усиливающее свойство правильности. Случайный вектор $\xi$ будем называть суперправильным; если для любого $h \in H$ распределение случайной величины $(\xi \mid h)$ инвариантно относительно всех вращений комплексной плоскости $\mathbf{C}^{1}$. Это равносильно тому, что распределение случайных величин $(\xi \mid h)$ и $\lambda(\xi \mid h)$ одинаковы для каждого $h \in H$ и для любого комплексного числа $\lambda$, по модулю равного единице.

В соответствии с общим определением, характеристическая функция случайной величины $(\xi \mid h)$ определяется равенством

$$
\chi_{(\xi \mid h)}(z)=\operatorname{E} \exp \{i \operatorname{Re}(\xi \mid h) \bar{z}\}, \quad z \in \mathbf{C}^{1}
$$

Напомним также, что характеристический функционал случайного вектора $\xi$ определяется соотношением

$$
\chi_{\xi}(h)=\operatorname{E} \exp \{i \operatorname{Re}(\xi \mid h)\}, \quad h \in H .
$$

Из равенств (11) и (12) следует, что для любых $z \in \mathbf{C}^{1}$ и $h \in H$

$$
\chi_{(\xi \mid h)}(z)=\chi_{\xi}(z h)
$$

Аналогичным образом легко показать, что свойство суперправильности случайного вектора $\xi$ можно выразить равенством $\chi_{\xi}(h)=\chi_{\xi}(\lambda h)$ 
для всех $h \in H$ и $\lambda \in \mathbf{C}^{\mathbf{1}},|\lambda|=1$. Это равенство означает инвариантность распределения случайного вектора $\xi$ относительно специального (довольно узкого) класса унитарных преобразований пространства $H$, именно, умножений на комплексные числа, модули которых равны единице.

В одномерном случае $\left(H=\mathbf{C}^{1}\right)$ суперправильность случайной величины $\zeta$ означает инвариантность ее распределения относительно умножения ее на $\lambda,|\lambda|=1$, или, другими словами, инвариантность относительно всех врапцений в $\mathbf{R}^{2}$ распределения двумерного вектора $\left(\zeta^{\prime}, \zeta^{\prime \prime}\right)$, составленного из вещественной и мнимой частей $\zeta$.

Заметим, что определение суперправильности применимо к любым случайным векторам в $H$, в то время как огределение правильности имеет смысл только в предположении существования ковариационного оператора. Поэтому сравнивать эти два свойства можно только для случайных векторов, имеюших слабый второй порядок.

Теорема 4. Пусть $\xi-$ суперправильный случайный вектор со значениями в комплексном гильбертовом пространстве Н. Тогда если $\xi$ имеет слабый второй порядок, то $\xi$ является правильным случайным вектором $и \mathrm{E} \xi=0$.

Д ок а 3 а т е л ь с т в о. Распределения случайных векторов $\xi$ и $\lambda \xi$ одинаковы для всех $\lambda \in \mathbf{C}^{1},|\lambda|=1$. Для наших пелей достаточна инвариантность распределения $\xi$ относительно умножения только на $\lambda=$ $i$. Действительно, в этом случае $K_{\left(\xi^{\prime}, \xi^{\prime \prime}\right)}=K_{\left(-\xi^{\prime \prime} \xi^{\prime}\right)}$. Следовательно, в силу соотношения (9) имеем $B_{\xi^{\prime}}=B_{-\xi^{\prime \prime}}$ и $T_{\xi^{\prime} \xi^{\prime \prime}}=T_{-\xi^{\prime \prime} \xi^{\prime}}$, т.е. $B_{\xi^{\prime}}=B_{\xi^{\prime \prime}}$ и $T_{\xi^{\prime} \xi^{\prime \prime}}^{*}=-T_{\xi^{\prime} \xi^{\prime \prime}}$. А это означает правильность случайного вектора $\xi$. Далее, $\mathbf{E}(\xi \mid h)=\mathbf{E}(i \xi \mid h)$ для всех $h \in H$, т.е. $\mathbf{E} \xi=0$.

3 а м е ч а н и е. Если $\xi$ имеет слабый второй порядок и распределение $\xi$ инвариантно относительно умножения $\xi$ на $i$, то $\xi$ - правильный случайный вектор и, кроме того, $\mathbf{E} \xi=0$.

\section{§3. Примеры правильных и суперправильных случайных векторов. Гауссовские случайные векторы}

Мы опишем два класса правильных и суперправильных случайных векторов. Первый из них более важный, второй показывает, что первый не исчерпывает всех возможностей. Пусть $L(H)$, как и выше, обозначает совокупность всех линейных непрерывных отображений из $H$ в $H$, а $\mathcal{B}$ обозначает наименьшую $\sigma$-алгебру подмножеств $L(H)$, относительно которой измеримы все функции $(A h \mid g): L(H) \longrightarrow \mathbf{C}^{1}$ при всех $h, g \in H$. Эквивалентным образом $\mathcal{B}$ можно определить как наименьшую $\sigma$-алгебру, относительно которой измеримы функции $\operatorname{tr} A N: L(H) \longrightarrow \mathbf{C}^{1}$ для всех ядерных операторов $N: H \rightarrow H$. Пусть, далее, $\Theta: \Omega \rightarrow L(H)-$ случайный линейный оператор в том смысле, что отображение $\Theta$ (линейно и) измеримо относительно $\sigma$-алгебры $\mathcal{B}$ в $L(H)$, т.е. числовые функции $(\Theta h \mid g)$ в $\Omega$ измеримы для всех $h, g \in H$. Это равносильно тому, что для 
каждого $h \in H$ функция $\Theta h: \Omega \longrightarrow H$ измерима, т.е. является случайным вектором в $H$.

Нетрудно показать, что для любого случайного вектора $\xi$ со значениями в $H$ вектор $\Theta \xi$, как функция в $\Omega$, является измеримым, т.е. обычным случайным вектором в $H$. В самом деле, ввиду сепарабельности $H$ достаточно доказать слабую измеримость суперпозиции $\Theta \xi$, т.е. измеримость функций $(\Theta \xi \mid h)$ для всех $h \in H$. Но $(\Theta \xi \mid h)=\left(\xi \mid \Theta^{*} h\right)$ и дело сводится к очевидному факту - измеримости скалярного произведения двух случайных векторов в сепарабельном гильбертовом пространстве (слабая, а потому и сильная измеримость вектора $\Theta^{*} h$ есть простое следствие измеримости $\Theta$ относительно $\sigma$-алгебры $\mathcal{B}$ ).

Теорема 5. Пусть случайный вектор $\xi$ и случайный линейный оператор $\Theta$ взаимно независимы. Тогда:

(1) если случайный вектор $\xi$ правильный и случайный вектор $\Theta \xi$ имеет слабый второй порядок, то $\Theta \xi$ также правильный;

(2) если $\xi$ суперправильный, то $\Theta \xi$ также суперправильный.

Д о к а з а т е л ь с т в о. Обозначим через $\mu$ распределение случайного вектора $\xi$ и через $\nu$ - распределение случайного линейного оператора $\Theta$. Используя независимость $\xi$ и $\Theta$ и теорему Фубини, получаем:

$$
\begin{aligned}
\mathbf{E}(\Theta \xi \mid h)^{2} & =\int_{L(H)} d \nu(t) \int(t x, h)^{2} d \mu(x) \\
& =\int_{L(H)} d \nu(t) \int_{H}\left(x \mid t^{*} h\right)^{2} d \mu(x)=0
\end{aligned}
$$

так как внутренний интеграл есть нуль в силу утверждения (6) теоремы 1. Правильность случайного вектора $\Theta \xi$ следует теперь из того же утверждения. Стоит отметить, что такое же рассуждение приводит к равенству

$$
\left(K_{\Theta \xi} h \mid h\right)=\int_{L(H)}\left(t K_{\xi} t^{*} h \mid h\right) d \nu(t), \quad h \in H .
$$

Аналогичным образом, используя соотношение (12), получим

$$
\chi_{\Theta \xi}(h)=\int_{L(H)} \chi_{\xi}\left(t^{*} h\right) d \nu(t), \quad h \in H,
$$

и для доказательства утверждения (2) теоремы остается заметить, что $\chi_{\Theta \xi}(\lambda h)=\chi_{\Theta \xi}(h)$ для всех $h \in H$ и $\lambda \in \mathbf{C}^{1},|\lambda|=1$, как это видно из предполагаемой суперправильности $\xi$ (мы используем критерий суперправильности, основанный на равенстве (13) и приведенный сразу после этого равенства).

Пусть $\zeta_{1}, \zeta_{2}, \ldots$ - некоторая последовательность взаимно независимых комплексных случайных величин, центрированных своими математическими ожиданиями и таких, что $\sum\left|\zeta_{k}\right|^{2}<\infty$ почти наверное. Обозначим $\xi=\sum \zeta_{k} e_{k}$, где $\left(e_{k}\right)$ - некоторый ортонормированный базис в 
$H$. Элементарно можно показать, что если $\sum \mathrm{E}\left|\zeta_{k}\right|^{2}<\infty$ и все $\zeta_{k}-$ правильные случайные величины, то $\xi$ - правильный случайный вектор. Далее, имеем:

$$
\chi_{\xi}(h)=\prod_{k=1}^{\infty} \chi_{\zeta_{k}}\left[\left(e_{k} \mid h\right)\right], \quad h \in H,
$$

и поэтому если все случайные величины $\zeta_{k}$ суперправильны, то $\xi-$ суперправильный случайный вектор (согласно критерию, основанному на равенстве (13)).

Случайный вектор $\xi$ в комплексном гильбертовом пространстве $H$ называется гауссовским, если все случайные величины $(\xi \mid h), h \in H$, являются гауссовскими. Эта часть определения совершенно естественна и не вызывает сомнений. Однако комплексные гауссовские случайные величины можно определить двумя различными способами, и это дает два различных определения в гильбертовом случае (как в конечномерном, так и бесконечномерном). Эта двойственность аналогична двойственности при определении ковариационного оператора. При одном, широком, подходе комплексная случайная величина $\zeta$ рассматривается как двумерная со значениями в вещественном пространстве $\mathbf{R}^{2}$ и называется гауссовской, просто если соответствующая пара $\left(\zeta^{\prime}, \zeta^{\prime \prime}\right)$ имеет гауссовское распределение в $\mathbf{R}^{2}$. А при другом, узком, подходе дополнительно требуется взаимная независимость $\zeta^{\prime}$ и $\zeta^{\prime \prime}$ и равенство их дисперсий. Это связано с тем, что за основу определения берется в этом подходе равенство (предполагается, что $\mathbf{E} \zeta=0$ )

$$
\chi_{\zeta}(z)=\exp \left\{-\frac{1}{4}|z|^{2} \mathbf{E}|\zeta|^{2}\right\}, \quad z \in \mathbf{C}^{1},
$$

обобщающее аналогичное равенство в вещественном случае. В самом деле, элементарно можно показать, что равенство (15) эквивалентно выполнению следующих условий: пара $\left(\zeta^{\prime}, \zeta^{\prime \prime}\right)$ - гауссовская в $\mathbf{R}^{2}, \mathbf{E} \zeta^{\prime}=$ $\mathbf{E} \zeta^{\prime \prime}=0, \mathbf{E} \zeta^{\prime} \zeta^{\prime \prime}=0$ и $\mathbf{E}\left(\zeta^{\prime}\right)^{2}=\mathbf{E}\left(\zeta^{\prime \prime}\right)^{2}$. Если $\mathbf{E}\left(\zeta^{\prime}\right)^{2}=\mathbf{E}\left(\zeta^{\prime \prime}\right)^{2}=1$, то соответствующую гауссовскую случайную величину $\zeta_{0}$ естественно назвать стандартной. Имеем $\chi_{\zeta_{0}}(z)=e^{-|z|^{2} / 2}$ и если за определение берется равенство (15), то все центрированные гауссовские случайные величины представляются как $a \zeta_{0}$ с некоторым комплексным коэффициентом $a$. Равенство $\zeta=a \zeta_{0}+b, a, b \in \mathbf{C}^{1}$, дает представление всех гауссовских случайных величин в узком смысле, т.е. в определении (15).

Случайный вектор $\xi$ в комплексном гильбертовом пространстве $H$ назовем гауссовским в широком (соответственно, узком) смысле, если все случайные величины $(\xi \mid h), h \in H$, являются гауссовскими в широком (соответственно, узком) смысле.

Теорема 6. Пусть $\xi-$ случайный вектор со значениями в комплексном гильбертовом пространстве $H, \xi=\xi^{\prime}+i \xi^{\prime \prime} u h=h^{\prime}+i h^{\prime \prime}-$ правильные разложения векторов $\xi$ и $h, u \hat{\xi}=\left(\xi^{\prime}, \xi^{\prime \prime}\right), h=\left(h^{\prime}, h^{\prime \prime}\right)-$ со- 
ответствующие векторы в $H_{\mathbf{R}} \oplus H_{\mathbf{R}}$. Пусть $\xi$ имеет слабый второй порядок $и \mathrm{E} \xi=0$. Тогда:

(1) $\xi$ является гауссовским в иироком смысле случайным вектором в том и только том случае, если $\hat{\xi}$ есть гауссовский случайный вектор в $H_{\mathbf{R}} \oplus H_{\mathbf{R}} ;$ в этом случае

$$
\chi_{\varepsilon}(h)=\exp \left\{-\frac{1}{2} \mathbf{E}\left[\left(\xi^{\prime} \mid h^{\prime}\right)+\left(\xi^{\prime \prime} \mid h^{\prime \prime}\right)\right]^{2}\right\}=\exp \left\{-\frac{1}{2}\left\langle K_{\hat{\xi}} \widehat{h} \mid \widehat{h}\right\rangle\right\}
$$

где $K_{\hat{\xi}}$ определяется равенством (9);

(2) छ является гауссовским в узком смысле случайным вектором $ө$ том и только том случае, если

$$
\chi_{\xi}(h)=\exp \left\{-\frac{1}{4} \mathbf{E}|(\xi \mid h)|^{2}\right\}=\exp \left\{-\frac{1}{4}\left(K_{\xi} h \mid h\right)\right\}, \quad h \in H,
$$

где $K_{\xi}$ определяется соотношением (6) (равенство (8) дает матрицу. $\widehat{K}_{\xi}$, соответствуюиую оператору $\left.K_{\xi}\right)$;

(3) гауссовский в узком смьсле случайный вектор является гауссовским в иироком смысле и правильным; обратно, гауссовский в иироком смысле правильный случайный вектор является гауссовским $u$ в узком смысле; таким образом, для правильных случайных векторов оба определения совпадают.

Д о к а 3 а т е л ь с т в о. Пусть $h-$ произвольный вектор из $H$ и $h^{\prime}+i h^{\prime \prime}$ - его правильное разложение. Обозначим $u_{1}=\left(\xi^{\prime} \mid h^{\prime}\right)+\left(\xi^{\prime \prime} \mid h^{\prime \prime}\right)$ и $u_{2}=\left(\xi^{\prime \prime} \mid h^{\prime}\right)-\left(\xi^{\prime} \mid h^{\prime \prime}\right)$. Для любых вещественных $\alpha$ и $\beta$ имеем $\alpha u_{1}+$ $\beta u_{2}=\langle\hat{\xi} \mid \hat{g}\rangle$, где $\widehat{g}=\left(\alpha h^{\prime}-\beta h^{\prime \prime}, \beta h^{\prime}+\alpha h^{\prime \prime}\right)$, и поэтому случайный вектор $\left(u_{1}, u_{2}\right)$ имеет гауссовское распределение в $\mathbf{R}^{2}$, если $\hat{\xi}-$ гауссовский вектор в $H_{\mathbf{R}} \oplus H_{\mathbf{R}}$. Обратное тривиально, так как $(\hat{\xi} \mid \widehat{h})=\operatorname{Re}(\xi \mid h)$. Далее,

$$
\begin{aligned}
\chi_{\varepsilon}(h) & =\mathbf{E} \exp \{i \operatorname{Re}(\xi \mid h)\}=\mathbf{E} \exp \{i\langle\hat{\xi} \mid \widehat{h}\rangle\}=\chi_{\varepsilon}(\widehat{h}) \\
& =\exp \left\{-\frac{1}{2}\left\langle K_{\hat{\xi}} \widehat{h} \mid \widehat{h}\right\rangle\right\}
\end{aligned}
$$

Докажем утверждение (2) теоремы. Равенство (17) для гауссовских в узком смысле случайных векторов следует из равенства (13) для $z=1$, равенства (15) для $\zeta=(\xi \mid h), z=1$, и равенства (5), определяющего ковариационный оператор $K_{\xi}$. Обратно, если (17) выполнено и $\zeta$ обозначает $(\xi \mid h)$, то $\chi_{\zeta}(z)$ имеет вид (15) согласно равенствам (13) и (5).

Прямое утверждение (3) есть следствие утверждений (4) или (5) теоремы 1. Обратное утверждение также вытекает из теоремы 1.

Случайный вектор $(\xi, \eta)$ в гильбертовой прямой сумме $H \oplus H$ будет гауссовским в широком смысле в том и только том случае, если 
$\left(\xi^{\prime}, \xi^{\prime \prime}, \eta^{\prime}, \eta^{\prime \prime}\right)$ будет гауссовским случайным вектором в вешественном пространстве $H_{\mathbf{R}}^{\mathbf{4}}=H_{\mathbf{R}} \oplus H_{\mathbf{R}} \oplus H_{\mathbf{R}} \oplus H_{\mathbf{R}}$, т.е. если

$$
\chi_{(\xi, \eta)}(h, g)=\exp \left\{-\frac{1}{2} \mathbf{E}\left[\left(\xi^{\prime} \mid h^{\prime}\right)+\left(\xi^{\prime \prime} \mid h^{\prime \prime}\right)+\left(h^{\prime} \mid g^{\prime}\right)+\left(h^{\prime \prime} \mid g^{\prime \prime}\right)\right]^{2}\right\} \text {. }
$$

Это равенство можно, очевидно, переписать как

$$
\chi_{(\xi, \eta)}(h, g)=\exp \left\{-\frac{1}{2}\left\langle K_{\hat{\xi}} \widehat{h} \mid \widehat{h}\right\rangle+\left\langle K_{\widehat{\eta}} \widehat{g} \mid \widehat{g}\right\rangle+2\left\langle T_{\hat{\xi} \hat{\eta}} \widehat{h} \mid \widehat{g}\right\rangle\right\},
$$

rде

$$
T_{\hat{\xi} \widehat{\eta}}=\left\|\begin{array}{cc}
T_{\xi^{\prime} \eta^{\prime}} & T_{\xi^{\prime \prime} \eta^{\prime}} \\
T_{\xi^{\prime} \eta^{\prime \prime}} & T_{\xi^{\prime \prime} \eta^{\prime \prime}}
\end{array}\right\|
$$

Как было отмечено перед формулировкой теоремы 3 , некоррелированность $\xi$ и $\eta$ (т.е. условие $T_{\xi \eta}=0$ ) дает не условие $T_{\hat{\xi} \hat{\eta}}=.0$, а всего лишь $T_{\xi^{\prime} \eta^{\prime}}+T_{\xi^{\prime \prime} \eta^{\prime \prime}}=0$ и $T_{\xi^{\prime} \eta^{\prime}}-T_{\xi^{\prime \prime} \eta^{\prime}}=0$. Поэтому, если гауссовость понимать в широком смысле, некоррелированность компонент не влечет их независимости. Однако теорема 3 спасает нашу интуицию, правда ценой уступки в отношении определения гауссовости. Вместе с тем это дает дополнительное соображение в пользу такого определения гауссовского случайного вектора.

Теорема 7. Пусть $(\xi, \eta)$ - аауссовский в узком смысле случайный вектор в $H \oplus H$. Тогда следуюшие условия эквивалентны:

(1) $\xi$ и өзаимно некоррелированны,

(2) $\xi$ и $\eta$ взаимно независимы,

(3) $T_{\xi^{\prime} \eta^{\prime}}=T_{\xi^{\prime \prime} \eta^{\prime \prime}}=T_{\xi^{\prime} \eta^{\prime \prime}}=T_{\xi^{\prime \prime} \eta^{\prime}}=0$

3 а м е ч н и е. Простой конкретный пример гауссовского в широком смысле случайного вектора $(\xi, \eta)$ с некоррелированными, но зависимыми координатами $\xi$ и $\eta$ можно дать даже для одномерного случая $H=\mathbf{C}^{1}$; достаточно взять пример из замечания к теореме 3 , предполагая, что $\left(\xi^{\prime}, \xi^{\prime \prime}\right)$ - совместно гауссовская пара вещественных случайных величин.

\section{§4. Почти наверное ортогональность случайных векторов}

В заметке [2] мы сформулировали условия почти наверное ортогональности двух случайных векторов $\xi_{1}$ и $\xi_{2}$ в вещественном гильбертовом пространстве (см. также работы [3] и [4], которые содержат доказательства). Несколько позже мы приведем здесь этот результат для удобства ссылки на него.

Пусть $\mathbf{E} \xi_{i}=0$ и $B_{i}(i=1,2)$ обозначает ковариационный оператор случайного вектора $\xi_{i}, T$ - оператор взаимной ковариации $\xi_{1}$ и $\xi_{2}$.

Теорема 8. $\mathrm{P}\left\{\left(\xi_{1} \mid \xi_{2}\right)=0\right\}=1$, если выполнены условия

$$
T^{*} B_{1}=-B_{1} T, \quad T B_{2}=-B_{2} T^{*}, \quad T^{2}=-B_{2} B_{1} .
$$


Обратно, почти наверное ортогональность $\xi_{1}$ и $\xi_{2}$ влечет выполнение условий (19), если дополнительно предположить, ито носитель распределения пары $\left(\xi_{1}, \xi_{2}\right)$ есть линейное многообразие.

Пусть $\xi$ и $\eta$ обозначают, как и раньше, случайные векторы со значениями в комплексном гильбертовом пространстве $H$. Всюду ниже будет предполагаться, что $\mathbf{E} \xi=\mathbf{E} \eta=0$.

В цитированных выше работах показано, что в вешественном случае система операторных уравнений (19) имеет нетривиальные решения и существуют даже невырожденные случайные векторы (т.е. такие, что $\left.B_{i} x=0 \Longrightarrow x=0 ; i=1,2\right)$, которые взаимно ортогональны с вероятностью 1. Здесь мы покажем, что в комплексном случае дело обстоит иначе: почти наверное ортогональность компонент правильной пары с линейным носителем возможна лишь в тривиальном случае, когда их значения принадлежат взаимно ортогональным подпространствам пространства $H$.

Теорема 9. Пусть $(\xi, \eta)-$ - равильный случайный вектор в гильбертовои прямой сумме $H \otimes H$ двух сепарабельных комплексных аильбертовых пространств $H$. Пусть, далее, $(\xi, \eta)$ имеет линейный носиmель $и \mathrm{E} \xi=\mathrm{E} \eta=0$.

Тогда если $\mathbf{P}\{(\xi \mid \eta)=0\}=1$, то существуют взаимно ортогональные подпространства $H_{1}$ и $H_{2}$ пространства $H$ такие, что $\xi \in H_{1}$ $u \in \in H_{2}$ с вероятностью 1 .

Дока за тельст в о. Пусть $\xi=\xi^{\prime}+i \xi^{\prime \prime}$ и $\eta=\eta^{\prime}+i \eta^{\prime \prime}-$ правильные разложения векторов $\xi$ и $\eta$, а $\hat{\xi}=\left(\xi^{\prime}, \xi^{\prime \prime}\right)$ и $\widehat{\eta}=\left(\eta^{\prime}, \eta^{\prime \prime}\right)$, соответствуюшие им случайные векторы в $H_{\mathbf{R}} \otimes H_{\mathbf{R}}$. Имеем

$$
(\xi \mid \eta)=\left(\xi^{\prime} \mid \eta^{\prime}\right)+\left(\xi^{\prime \prime} \mid \eta^{\prime \prime}\right)+i\left[\left(\xi^{\prime \prime} \mid \eta^{\prime}\right)-\left(\xi^{\prime} \mid \eta^{\prime \prime}\right)\right]
$$

и поэтому условие $\mathbf{P}\{(\xi \mid \eta)=0\}=1$ равносильно двум условиям

$$
\mathbf{P}\{(\hat{\xi} \mid \widehat{\eta})=0\}=1 \quad \text { и } \quad \mathbf{P}\{(\hat{\xi} \mid J \widehat{\eta})=0\}=1,
$$

где $J$ есть оператор, определенный матрицей (2). Очевидно, что отображение $(\widehat{x}, \widehat{y}) \longmapsto\left(x^{\prime}+i x^{\prime \prime}, y^{\prime}+i y^{\prime \prime}\right)$ пространства $H_{\mathrm{R}}^{4}$ на $H \otimes H$, а также отображение $(\widehat{x}, \widehat{y}) \longmapsto(\widehat{x}, J \widehat{y})$ пространства $H_{\mathbf{R}}^{4}$ на себя представляют собой линейные гомеоморфизмы. Поэтому из линейности носителя $(\xi, \eta)$ вытекает линейность носителей случайных векторов $(\hat{\xi}, \widehat{\eta})$ и $(\hat{\xi}, J \widehat{\eta})$, и, согласно соотношениям (20) и теореме 8 , должны выполняться условия (19) для двух наборов: $B_{1}=K_{\hat{\xi}}, B_{2}=K_{\hat{\eta}}, T=T_{\hat{\xi} \hat{\eta}}$ и $B_{1}=K_{\hat{\xi}}, B_{2}=K_{J \hat{\eta}}$, $T=T_{\hat{\xi}(J \widehat{\eta})}$. Эти операторы определяются, согласно общим определениям (7), равенствами

$$
\left\langle K_{\hat{\xi}} \widehat{h} \mid \widehat{h}\right\rangle=\mathbf{E}\langle\hat{\xi} \mid \widehat{h}\rangle^{2}, \quad\left\langle T_{\hat{\xi} \widehat{\eta}} \widehat{h} \mid \widehat{g}\right\rangle=\mathbf{E}(\hat{\xi} \mid \widehat{h})(\widehat{\eta} \mid \widehat{g})
$$

аналогично определяются $K_{\widehat{\eta}}$ и $T_{\hat{\xi}(J \widehat{\eta})}$. Непосредственно из этих определений получаем, что $K_{J \widehat{\eta}}=J K_{\widehat{\eta}} J^{*}$ и $T_{\hat{\xi}(J \widehat{\eta})}=J T_{\hat{\xi} \widehat{\eta}}$. Используем третье 
равенство в $(19)$ для обоих наборов $\left(K_{\hat{\xi}}, K_{\widehat{\eta}}, T_{\hat{\xi} \widehat{\eta}}\right)$ и $\left(K_{\hat{\xi}}, J K_{\widehat{\eta}} J^{*}, J T_{\hat{\xi} \hat{\eta}}\right)$. Получим

$$
T_{\hat{\xi} \hat{\eta}}^{2}=-K_{\hat{\eta}} K_{\hat{\xi}}, \quad T_{\hat{\xi} \hat{\eta}} J T_{\hat{\xi} \hat{\eta}}=K_{\widehat{\eta}} J K_{\hat{\xi}}
$$

По условию теоремы случайный вектор $(\xi, \eta)$ правильный. Поэтому, согласно теореме $2, T_{\xi^{\prime} \eta^{\prime}}=T_{\xi^{\prime \prime} \eta^{\prime \prime}}$ и $T_{\xi^{\prime} \eta^{\prime \prime}}=-T_{\xi^{\prime \prime} \eta^{\prime}}$. Используя эти равенства, несложно показать, что $T_{\hat{\xi} \widehat{\eta}} J=J T_{\hat{\xi} \widehat{\eta}}$. Правильность случайного вектора $(\xi, \eta)$ влечет правильность каждой из компонент $\xi$ и $\eta$ (теорема 2). Поэтому, по теореме $6(3)$, оператор $J$ коммутирует также и с операторами $K_{\hat{\xi}}$ и $K_{\widehat{\eta}}$ (достаточно было бы свойства коммутативности с одним из них). Следовательно, равенства (21) дают соотношение $K_{\widehat{\eta}} K_{\hat{\xi}}=0$, из которого следует взаимная ортогональность подпространств $\bar{K}_{\hat{\xi}}\left(H_{\mathbf{R}} \otimes H_{\mathbf{R}}\right)$ и $\bar{K}_{\widehat{\eta}}\left(H_{\mathbf{R}} \otimes H_{\mathbf{R}}\right)$ пространства $\left(H_{\mathbf{R}} \otimes H_{\mathbf{R}}\right)$ (замыканий образов при этих отображениях). Легко сообразить также, что всегда $\mathbf{P}\left(\xi \in \bar{K}_{\xi}(H)\right\}=1$ и поэтому за $H_{1}$ и $H_{2}$ можно взять образы подпространств $\bar{K}_{\hat{\xi}}\left(H_{\mathbf{R}} \otimes H_{\mathbf{R}}\right)$ и $\bar{K}_{\hat{\eta}}\left(H_{\mathbf{R}} \otimes H_{\mathbf{R}}\right)$ при естественном изометрическом изоморфизме $\left(H_{\mathbf{R}} \otimes H_{\mathbf{R}}\right)$ на $H$ (точнее, на $\left.H_{0}\right)$.

Следствие. Компоненты $\xi$ и $\eta$ гауссовского (в узком смысле) случайного вектора $(\xi, \eta)$ в $H \otimes H$ могут быть почти наверное взаинно ортогональны лишь в том случае, если они с вероятностью 1 принадлежат взаимно ортогональньм подпространствам пространства $H$.

3 а м е ч а н и е. Предположение правильности вектора $(\xi, \eta)$ в теореме 9 и в следствии к ней существенно. Можно дать пример даже гауссовских в узком смысле случайных векторов $\xi$ и $\eta$, которые образуют гауссовскую в широком смысле пару $(\xi, \eta)$ и взаимно ортогональны с вероятностью 1 , хотя $K_{\widehat{\eta}} K_{\hat{\xi}}$ обращается в нуль только в нуле. Вот этот пример: пусть $\xi^{\prime}$ н $\xi^{\prime \prime}$ - взаимно независимые центрированные гауссовские случайные векторы в $H_{\mathbf{R}}$ и $\xi=\xi^{\prime}+i \xi^{\prime \prime}, \eta=V \xi^{\prime}+i V \xi^{\prime \prime}$, где $V-$ косоэрмитов линейный оператор $\left(V^{*}=-V\right)$.

\section{§5. Шёнберговские случайные векторы}

В этом параграфе мы продолжаем исследование суперправильных случайных векторов, отправляясь от соотношения (14). Пусть, как и выше, $\Theta: \Omega \longrightarrow L(H)$ - случайный линейный оператор, и пусть $\xi$ обозначает сейчас конкретный суперправильный случайный вектор, именно, гауссовский в узком смысле вектор со средним нуль и ковариационным оператором $K_{\xi}$. Хорошо известно, что если $\xi$ - гауссовский случайный вектор в вещественном гильбертовом пространстве, то $\mathbf{E}\|\xi\|^{2}=$ $\operatorname{tr} B_{\xi}<\infty$. Поэтому в рассматриваемом нами комплексном случае имеем $\mathbf{E}\|\xi\|^{2}=\mathbf{E}\left\|\xi^{\prime}\right\|^{2}+\mathbf{E}\left\|\xi^{\prime \prime}\right\|^{2}=2 \operatorname{tr} B$, где $B$ - общий ковариашионный оператор случайных векторов $\xi^{\prime}$ и $\xi^{\prime \prime}$. С другой стороны,

$$
\mathbf{E}\|\xi\|^{2}=\mathbf{E}\left\|\Sigma\left(\xi \mid e_{k}\right) e_{k}\right\|^{2}=\mathbf{E} \Sigma\left|\left(\xi \mid e_{k}\right)\right|^{2}=\Sigma\left(K_{\xi} e_{k} \mid e_{k}\right)=\operatorname{tr} K_{\xi}
$$


Следовательно, $\operatorname{tr} K_{\xi}=2 \operatorname{tr} B$, и класс ковариационных операторов $K_{\xi}$ всех гауссовских случайных векторов $\xi$ совпадает, как и в вешественном случае, с классом всех линейных самосопряженных положительных операторов из $H$ в $H$, имеющих конечный след, т.е. ядерных. Любой такой оператор $K$ и любое распределение $\nu$ на $L(H)$ порождает по равенству (14) характеристический функционал $\chi$ :

$$
\chi(h)=\chi(h ; K, \nu)=\int_{L(H)} \exp \left\{-\frac{1}{4}\left(t K t^{*} h \mid h\right)\right\} d \nu(t) .
$$

Пусть $\{K\}^{\prime}$ обозначает множество всех операторов из $L(H)$, которые перестановочны с $K$. Заметим, что $\{K\}^{\prime}$ есть алгебра Неймана, но для нас сейчас важно только то, что $\{K\}^{\prime}$ есть измеримое множество в $L(H)$.

Tеорема 10. (1) $\{K\}^{\prime} \in \mathcal{B}$;

(2) $\left\{t: t \in L(H), t Q t^{*}=Q\right\} \in \mathcal{B}$ для любого фиксированного операmopa $Q \in L(H)$.

Д о к а з а т л л с т в о. Эти утверждения следуют из замкнутости указанных множеств в слабой операторной топологии. Но они могут быть доказаны также и элементарно. Для доказательства утверждения (1) используем следующее простое описание множества $\{K\}^{\prime}$, имеющее также самостоятельный интерес. Пусть $s_{1}, s_{2}, \ldots$ - последовательность различных собственных значений оператора $K$ и $m_{j}$ кратность собственного значения $s_{j}(j=1,2, \ldots)$. Ввиду ядерности и положительности оператора $K$, каждое $m_{j}$ есть положительное целое число и $\sum_{j=1}^{\infty} m_{j} s_{j}=\operatorname{tr} K<\infty$. Ясно, что матрица оператора $K$ в базисе собственных векторов $\left(e_{j}\right)$ этого же оператора может быть записана как блочно-диагональная с матрицами $s_{1} I_{m_{1}}, s_{2} I_{m_{2}}, \ldots$ на диагонали, $I_{m_{j}}-$ единичная матрица порядка $m_{j}$. Пусть, как это принято, $\operatorname{Mat}_{m_{j}}\left(C^{1}\right)$ обозначает множество всех квадратных комплексных матриц порядка $m_{j}$. Нетрудно сообразить, что совокупность матриц, соответствуюших операторам из $\{K\}^{\prime}$ в базисе $\left(e_{j}\right)$, совпадает с прямой суммой

$$
\underset{k+1}{\infty} \operatorname{Mat}_{m_{k}}\left(\mathbf{C}^{1}\right)
$$

Эта сумма есть совокупность всех блочно-диагональных матриц со всевозможными квадратными комплексными матрицами порядков $m_{1}$, $m_{2}, \ldots$ на диагонали. Обозначим через $O_{1}$ множество пар индексов $(i, j)$, соответствующих элементам этих квадратных матриц (т.е. множество мест, занятых элементами этих матриц), и пусть $\mathrm{O}_{2}$ есть множество всех остальных индексов. Очевидно, что

$$
\bigoplus_{k+1}^{\infty} \operatorname{Mat}_{m_{k}}\left(\mathbf{C}^{1}\right)=\bigcap_{(i, j) \in O_{2}}\left\{A:\left(A e_{i} \mid e_{j}\right)=0\right\}
$$

и остается только заметить, что $\left\{A:\left(A e_{i} \mid e_{j}\right)=0\right\} \in B$ для каждой пары индексов $(i, j)$ и множество $\mathrm{O}_{2}$ счетно. 
Докажем теперь утверждение (2) (в доказательстве этого утверждения ортонормированный базис $\left(e_{j}\right)$ может быть произвольным). Очевидно, что функции $f_{m, n}(A): L(H) \longrightarrow \mathbf{C}^{1}$, определяемые. соотношениями

$$
f_{m, n}(A)=\sum_{i=1}^{\infty}\left[\sum_{j=1}^{\infty}\left(A e_{j} \mid e_{m}\right)\left(Q e_{i} \mid e_{j}\right)\right] \overline{\left(A e_{j} \mid e_{n}\right)}
$$

измеримы при всех значениях чисел $m$ и $n$. Поэтому

$$
\bigcap_{m, n=1}^{\infty}\left\{A: f_{m n}(A)=\left(Q e_{n} \mid e_{m}\right)\right\} \in \mathcal{B} .
$$

Но это пересечение как раз и есть множество всех тех операторов $A \in$ $L(H)$, которые удовлетворяют условию $A Q A^{*}=Q$.

Доказательство теоремы закончено.

3 а м е ч а н и е. Если в качестве $Q$ взять тождественный оператор в $H$, то утверждение (2) даст измеримость множества операторов, имеющих изометрические сопряженные. Аналогично можно показать измеримость множества изометрических операторов. Следовательно, измеримо также множество унитарных операторов (как пересечение двух предыдуших множеств).

Возьмем теперь пересечение любого из этих множеств с множеством $\{K\}^{\prime}$, структура которого описывается в соотношении (23). Получим совокупность операторов, имеющих в базисе собственных векторов оператора $K$ блочно-диагональные матрицы с унитарными операторами порядков $m_{1}, m_{2}, \ldots$ на диагонали. Следовательно, справедливо следующее следствие из доказанной теоремы.

\section{Следствие.}

$$
\bigoplus_{k+1}^{\infty} U\left(\mathbf{C}^{m_{k}}\right) \in \mathcal{B}
$$

где $U\left(\mathbf{C}^{m_{k}}\right)$ - множество унитарных операторов 6 комплексном $m_{k}$ мерном евклидовом пространстве $\mathrm{C}^{m_{k}}$.

Перейдем, наконец, к последнему вопросу --. к описанию одного класса суперправильных случайных векторов, которые мы называем шёнберговскими. Чтобы мотивировать это название, напомним некоторые известные факты. В 1938 году И. Дж. Шёнберг нашел следующую формулу, описывающую все изометрически инвариантные (зависящие только от нормы элемента) непрерывные положительно определенные функционалы $\varphi$ в вещественном бесконечномерном гильбертовом пространстве $H_{\mathbf{R}}$ :

$$
\varphi(x)=\int_{\mathbf{R}^{1}} \exp \left\{-\frac{s^{2}}{2}\|x\|^{2}\right\} d \nu_{\mathbf{R}}(s)
$$


где $\nu_{\mathbf{R}}$ - произвольная конечная борелевская мера на числовой оси, которую можно считать вероятностной мерой, если нормировать функционалы условием $\varphi(0)=1$ (более подробно о теореме Шёнберга см. [1, гл. 4, § 4]).

Пусть $S$ - произвольный линейный ограниченный самосопряженный положительный оператор. Будем считать, что $S$ невырожден (из равенства $S x=0$ следует, что $x=0$ ). Для справедливости теоремы Шёнберга полнота пространства не требуется. Поэтому, если в $H_{\mathbf{R}}$ ввести новое скалярное произведение с помощью оператора $S$, то формула Шёнберга с заменой $\|x\|^{2}$ на $(S x \mid x)$ даст следующий общий вид всех непрерывных положительно определенных функционалов в $H_{\mathbf{R}}$, зависяцих только от квадратичной формы $(S x \mid x)$ :

$$
\chi\left(x ; S, \nu_{\mathbf{R}}\right)=\int_{\mathbf{R}^{3}} \exp \left\{-\frac{s^{2}}{2}(S x \mid x)\right\} d \nu_{\mathbf{R}}(s) .
$$

Поясним, что выражение « $\chi$ зависит только от $(S x \mid x)$ означает следующее: если $(S x \mid x)=(S y \mid y)$, то $\chi(x)=\chi(y)$.

Для любой борелевской вероятностной меры $\nu_{\mathbf{R}}$ функционал (24) будет характеристическим функционалом некоторой вероятностной меры $\sigma\left(S, \nu_{\mathbf{R}}\right)$ в $H_{\mathbf{R}}$ в том и только том случае, если $S$ является ковариационным оператором гауссовской меры, т.е. дополнительно обладает свойством ядерности (имеет конечный след). Поэтому совокупность $\left\{\sigma\left(S, \nu_{\mathbf{R}}\right)\right\}$ таких мер для всех невырожденных гауссовских ковариационных операторов $S$ и борелевских вероятностных мер $\nu_{\mathbf{R}}$ есть множество всех «эллиптически контуированных» мер, т.е. мер, характеристические функционалы которых зависят только от $(S x \mid x)$. Мера $\sigma\left(S, \nu_{\mathbf{R}}\right)$ есть, очевидно, смесь по весовой мере $\nu_{\mathbf{R}}$ центрированных гауссовских распределений в $H_{\mathbf{R}}$ с ковариационными операторами $s^{2} S$. Легко также видеть, что каждая такая мера есть распределение случайного вектора $\theta_{\mathbf{R}} \xi$, где $\xi$ - центрированный гауссовский случайный вектор в $H_{\mathbf{R}} \mathbf{c}$ ковариационным оператором $S$ и $\theta_{\mathbf{R}}$ - не зависящая от $\xi$ вешественная случайная величина с распределением $\nu_{\mathbf{R}}$. Такие меры изучались в литературе под названием эллиптически контуированшых мер (см., например, [5]).

Случайные векторы со значениями в $H_{\mathbf{R}}$, имеющие распределения $\sigma\left(S, \nu_{\mathbf{R}}\right)$ для каких-либо $S$ и $\nu_{\mathbf{R}}$, будем называть шёнберговскими. Аналогичным образом, случайный вектор со значениями в комплексном гильбертовом Іространстве $H$ будем называть шёнберговским, если его характеристический функционал имеет вид

$$
\chi\left(h ; K, \nu_{\mathbf{C}}\right)=\int_{\mathbf{C}^{\mathbf{1}}} \exp \left\{-\frac{|\lambda|^{2}}{4}(K h \mid h)\right\} d \nu_{\mathbf{C}}(\lambda),
$$

где $K$ - ковариационный оператор некоторого гауссовского (в узком смысле) случайного вектора (см. (5) и (6)) и $\nu_{\mathbf{C}}-$ борелевская веро- 
ятностная мера на комплексной плоскости $\mathbf{C}^{\mathbf{1}}$. Легко видеть, что мера, соответствуюшая характеристическому функционалу (25), является смесью по весовой мере $\nu_{\mathrm{C}}$ центрированных гауссовских распределений в $H$ с ковариационными операторами $|\lambda|^{2} K$. Это равносильно тому, что каждая такая мера с параметрами $K$ и $\nu_{\mathrm{C}}$ есть распределение случайного вектора $\theta_{\mathbf{C}} \xi$, где $\xi-$ центрированный гауссовский случайный вектор в $H$ с ковариационным оператором $K$ и $\theta_{\mathbf{C}}$ - не зависящая от $\xi$ комплексная случайная величина с распределением $\nu_{\mathbf{C}}$.

Таким образом, шёнберговские меры в $H_{\mathbf{R}}$ и $H$ можно определить как смеси гауссовских мер, или, эквивалентным образом, как распределения случайных векторов $\theta_{\mathbf{R}} \xi$ и $\theta_{\mathbf{C}} \xi$. Здесь $\theta_{\mathbf{R}}$ и $\theta_{\mathbf{C}}-$ случайные величины. Если же вернуться к случайным линейным операторам $\Theta: H \rightarrow H$, введенным в §3, то получим, очевидно, формулу (22) для характеристического функционала распределения $\Theta \xi$, где $\xi$, как и раньше, есть гауссовский (в узком смысле) случайный вектор с ковариационным оператором $K$ (мера $\nu$ в (22) будет распределением $\Theta$ ). Поэтому случайный вектор в $H$ с характеристическим функционалом (22) можно назвать обобщенным шёнберговским случайным вектором. Его распределение, как и шёнберговские распределения (25) (для случая комплексных пространств) и (24) (для случая вещественных пространств) является смесью гауссовских распределений. Соответствуюшие ковариационные операторы в этом случае имеют вид $t K t^{*}, t \in L(H)$, а весовая мера $\nu$ задана на $\sigma$-алгебре $\mathcal{B}$ пространства $L(H)$.

Однако в общем случае характеристический функционал (22) необязательно «эллиптически контуирован», т.е. необязательно зависит только от $(K h \mid h)$, и, таким образом, соответствующие распределения не обладают этим свойством шёнберговских распределений.

Предположим теперь, что $\nu\left(\{K\}^{\prime}\right)=1$ (измеримость $\{K\}^{\prime}$ доказана в теореме 10). В этом частном случае получим формулу

$$
\chi(h ; K, \nu)=\int_{\{K\}^{\prime}} \exp \left\{-\frac{1}{4}\left(t t^{*} K h \mid h\right)\right\} d \nu(t),
$$

более похожую на формулу (25), которая соответствует еще более частному случаю, когда $\Theta$ есть произведение скалярной (комплексной) случайной величины на единичный оператор в $H$.

Заметим, что аналогичным образом можно ввести понятие обобщенного шёнберговского случайного вектора со значениями в вещественном гильбертовом пространстве $H_{\mathbf{R}}$. Для этого достаточно случайный линейный оператор $\Theta$ считать действующими в $H_{\mathbf{R}}$ и взять не зависящий от него гауссовский случайный вектор, принимающий значения в $H_{\mathbf{R}}$.

Формулы (22) и (26) можно интерпретировать следуюшим образом. Пусть через некоторый фильтр проходит гауссовский случайный вектор (сигнал) $\xi$ со средним нуль и невырожденным ковариапионным оператором $K$, и пусть прохождение сигнала через фильтр заключается в воз- 
действии на $\xi$ не зависящим от него случайным линейным оператором $\Theta$, имеющим распределение $\nu$. Поставим вопрос: при каких условиях сигнал проходит через такой фильтр без искажения его распределения, т.е. при каких условиях обобщенное шёнберговское распределение (22) или (26) есть гауссовское распределение с ковариационным оператором $K$ ? Ответ дается в следуюшей теореме, в первом утверждении которой рассматривается общий случай, а во втором - случай, когда распределение $\nu$ сосредоточено на $\{K\}^{\prime}$. В этом предположении, естественно, ответ более конструктивный.

Пусть, как и выше, $m_{1}, m_{2}, \ldots$ - кратности собственных значений оператора $K$ и $\{K\}^{\prime}$ - совокупность всех операторов из $L(H)$, которые коммутируют с $K$ (структура множества $\{K\}^{\prime}$ описывается соотношением (23)).

Теорема 11. (1) Необходимым и достаточным условием выполнения равенства

$$
\int_{L(H)} \exp \left\{-\frac{1}{4}\left(t K t^{*} h \mid h\right)\right\} d \nu(t)=\exp \left\{-\frac{1}{4}(K h \mid h)\right\}
$$

для всех $h \in H$ является условие

$$
\nu\left\{t: t \in L(H), t K t^{*}=K\right\}=1 \text {. }
$$

(2) Необходимым и достаточным условием выполнения равенства

$$
\int_{\{K\}^{\prime}} \exp \left\{-\frac{1}{4}\left(t t^{*} K h \mid h\right)\right\} d \nu(t)=\exp \left\{-\frac{1}{4}(K h \mid h)\right\}
$$

для всех $h \in H$ является равенство

$$
\nu\left(\underset{k=1}{\infty} U\left(\mathbf{C}^{m_{k}}\right)\right)=1
$$

Д о к а $з$ а т е л ь с т в о. (1) Обозначим через $\psi_{h}(t)$ подынтегральное выражение в (27). Подставляя в (27) вместо $h$ значение $\sqrt{2} h$, получим равенство

$$
\int \psi_{h}^{2}(t) d \nu(t)=\left[\int \psi_{h}(t) d \nu(t)\right]^{2} \quad \text { для всех } \quad h \in H
$$

Отсюда, ввиду сепарабельности $H$, элементарно следует соотношение

$$
\nu\left\{t: \psi_{h}(t)=\int \psi_{h}(t) d \nu(t) \text { для всех } h \in H\right\}=1,
$$

из которого легко получить условие (28) (напомним, что измеримость доказана в теореме 10(2)). Достаточность тривиальна. 
(2) Достаточность станет очевидной, если учесть структуру множества $\{K\}^{\prime}$. Докажем необходимость. В силу утверждения (1) теоремы и невырожденности оператора $K$, равенство (29) дает условие $\nu\left\{t: t \in\{K\}^{\prime}, t t^{*}=I\right\}=1$ ( $I$ - тождественный оператор). Свойство $t t^{*}=I$ для операторов в конечномерных пространствах равносильно свойству унитарности оператора. Поэтому выражение (23) показывает, что если $t \in\{K\}^{\prime}$ и $t t^{*}=I$, то матрица оператора $t$ в базисе собственных векторов оператора $K$ блочно-диагональна с произвольными унитарными матрицами порядков $m_{1}, m_{2}, \ldots$ на диагонали. Следовательно, условие (29) влечет (30) (напомним, что измеримость доказана в следствии из теоремы 10).

Доказанная теорема означает, что указанный выше фильтр с преобразователем $\Theta$ не меняет распределения входного гауссовского сигнала $\xi$ только в том случае, если почти все значения $\Theta$ принадлежат множеству операторов, оставляюших инвариантным распределение сигнала $\xi$. Это множество обычно считается слишком бедным. Возможно, это впечатление связано с несуществованием изометрически инвариантных мер в бесконечномерном пространстве. На самом деле, степень симметрии распределения $\xi$ не так уж незначительна. Соответственно, множество операторов, не меняюших распределения $\xi$, не так уж бедно. В предположении $\nu\left(\{K\}^{\prime}\right)=1$ это множество есть $\oplus U\left(\mathbf{C}^{m_{k}}\right)$, и оно тем богаче, чем больше имеется кратных собственных значений у ковариационного оператора $K$ и чем выше их кратности.

\section{СПИСОК ЛИТЕРАТУРЫ}

1. Вахания Н. Н., Тариеладзе В. И., Чобанян С. А. Вероятностные распределения в банаховых пространствах. М.: Наука, 1985, 368 с.

2. Вахания H. Н., Каяделахи Н. П. Об ортогональных случайных векторах в гильбертовом пространстве. - Докл. АН СССР, 1987, т. 294, № 3, с. 528-531.

3. Вахания H. Н., Канделаки Н. П. Ортогональные случайные векторы в гильбертовом пространстве. - Труды Ин-та вычисл. матем. АН ГССР, 1988, т. 28, № 1 , c. 11-37.

4. Vakhania $N$. Orthogonal random vectors and the Hurwitz-Radon-Eckmann theorem. - Proc. Georgian Acad. Sci. Math., 1993, v. 1, № 1, p. 109-125.

5. Crawford J. J. Elliptically contoured measures on infinite-dimensional Banach spaces. - Studia Mathematica, 1977, v. 60, № 1, p. 15-32.

6. Вахания H. H., Канделаки $H$. ПI. Спаривание случайных векторов и шёнберговские характеристические функционалы. - Труды Ин-та вычисл. матем. АН ГCCP, 1990, т. 29, № 1, c. 77-84. 